\title{
Neural Activation Profile Elicited by Cues Associated with the Anxiogenic Drug Yohimbine Differs from That Observed for Reward-Paired Cues
}

\author{
Brock E Schroeder', Craig A Schiltz ${ }^{2}$ and Ann E Kelley*,3 \\ 'Department of Psychology, Arizona State University, Tempe, AZ, USA; ${ }^{2}$ Neuroscience Training Program, University of Wisconsin-Madison, \\ Madison, WI, USA; ${ }^{3}$ Department of Psychiatry, Medical School, University of Wisconsin-Madison, Madison, WI, USA
}

\begin{abstract}
Cues associated with dangerous or rewarding outcomes can themselves elicit neural activation. Previous work in rats has shown that cues associated with morphine, cocaine, nicotine or palatable food can elicit enhanced expression of the immediate-early gene product Fos in discrete brain regions. Activation of the prefrontal cortex has been shown to be particularly prominent. Some studies have also shown prefrontal cortical activation following exposure to fear-inducing stimuli. To investigate the specificity of regional brain Fos activation, we treated rats with an anxiogenic drug, yohimbine $(2 \mathrm{mg} / \mathrm{kg}$, intraperitoneally (i.p.)), or water once per day for 10 consecutive days in a test environment distinct from their home cages. Yohimbine elicited a robust locomotor response that progressively sensitized over days. After a 4-day interval, rats were reintroduced to the paired environment, without drug treatment. Rats re-exposed to the environment where they had previously been treated with yohimbine showed conditioned increases in motor activity compared with controls. Fos expression was increased in several brain regions, including the basolateral amygdala, but was unchanged in prefrontal cortex, in contrast to what has been reported for rewarding drugs. These observations show a neural activation profile elicited by cues associated with the anxiogenic drug yohimbine and further support the hypothesis that prefrontal cortex has a specific role in reward expectancy. Neuropsychopharmacology (2003) 28, |4-21. doi:I0.1038/sj.npp. 1300007
\end{abstract}

Keywords: yohimbine; amygdala; prefrontal cortex; conditioning; classical; genes; fos; anxiety

\section{INTRODUCTION}

Environmental cues that become associated with specific experiences and events can themselves become powerful triggers of physiological responses and neural activation. These Pavlovian associations are of obvious adaptive value in recognizing potentially dangerous situations, as in the case of fear conditioning (Kandel et al, 2000; LeDoux, 2000). Environmental stimuli that have come to be associated with positive events can also serve an adaptive function, as in the case of salivation in Pavlov's dogs. Pavlovian associations have also been implicated as one potential factor leading to drug addiction and relapse (Wikler and Pescor, 1967; O'Brien et al, 1998; Self and Nestler, 1998). In an attempt to explore brain mechanisms underlying drug conditioning, we have previously shown that cues associated with either morphine, nicotine, or chocolate cause increases in neural activation within discrete brain regions, as measured by expression of the

* Correspondence: AE Kelley, Department of Psychiatry, 6001 Research Park Blvd. Madison, WI 53719, USA, Tel: +I 608262 I I23, Fax: + | 608265 3050, E-mail: aekelley@facstaff.wisc.edu

Received I October 200 I; revised 29 May 2002; accepted 3 June 2002 Online publication: 5 June 2002 at http://www.acnp.org/citations/ Npp060502320 immediate-early gene product Fos (Schroeder et al, 2000; Schroeder et al, 2001). Related work has shown a similar pattern for cocaine-related cues (Brown et al, 1992; Franklin and Druhan, 2000; Ciccocioppo et al, 2001). A common finding in each of these experiments has been neural activation in the prefrontal cortex following exposure to the drug- or food-paired environment. These data, as well as findings from imaging studies in human subjects (Grant et al, 1996; Maas et al, 1998; Childress et al, 1999), have led to the hypothesis that the prefrontal cortex mediates drug or reward expectancy, and that the emotional state associated with craving involves abnormal activation of this region.

In addition to the amygdala, which is commonly thought to be involved in fear-related behavior, several fear conditioning studies have also implicated the prefrontal cortex (Beck and Fibiger, 1995; Campeau et al, 1997). Other studies, however, report no activation of the prefrontal cortex in fear conditioning (Morrow et al, 1999). It is important to consider the specificity of this activation, given that the prefrontal cortex appears to be activated by cues associated with several different types of rewarding stimuli (Franklin and Druhan, 2000; Schroeder et al, 2000, 2001; Ciccocioppo et al, 2001). Is the prefrontal cortex specifically activated by positive and rewarding conditioned stimuli, or 
do cues associated with negative experiences also stimulate this important brain region?

Here we investigated the question of whether cues associated with a drug known to have aversive properties would elicit a similar pattern of Fos activation under the paradigm we have used previously to examine Fos expression associated with rewarding stimuli. Instead of pairing an injection of a rewarding drug with a specific environment, an environment was paired with an injection of yohimbine, an anxiogenic $\alpha-2$ adrenergic receptor antagonist. Yohimbine causes an increase in the firing of central nervous system noradrenergic neurons and, in humans, increases subjective reports of anxiety (Charney et al, 1983; Uhde et al, 1984; Sanger, 1988). Similarly, in animal studies, yohimbine has been shown in multiple paradigms to be apparently anxiogenic, causing conditioned place aversion, potentiated startle, decreased social interaction and decreased exploration (Davis et al, 1979; Pellow et al, 1985; Chopin et al, 1986; File, 1986; Bhattacharya et al, 1997).

Our goals in this study were two-fold: first, to determine what brain regions are activated by environmental cues associated with an aversive drug experience and, second, to compare this regional neural activation profile to that observed following exposure to reward-paired cues.

\section{METHODS}

\section{Subjects and Housing}

A total of 20 male Sprague-Dawley rats (Harlan, Madison, WI) were used in this study. Pairs of rats were housed in clear plastic cages in an animal colony. Food and water were available at all times. Lighting in the animal colony was on a 12-h light/dark cycle (lights on $0700-1900 \mathrm{~h}$ ). Rats were handled upon arrival in the laboratory and daily thereafter, to minimize handling stress during testing. All efforts were made to minimize both the suffering and the number of animals used. All animal care was in strict accordance with our Institutional Animal Care and Use Committee guidelines.

\section{Behavioral Testing}

The environment-specific yohimbine administrations were conducted in clear, polycarbonate activity cages (approximately $48 \times 25 \times 20 \mathrm{~cm}(19 \times 10 \times 8$ in $)$; San Diego Instruments), in a separate testing room from the animal colony. Four infrared photobeams spaced at regular intervals along the bottom length of the cages recorded both horizontal activity (any beam being broken) and ambulatory activity (consecutive breaks of adjacent beams). Eight photobeams spaced at regular intervals along the top width of the cages recorded rearing (vertical activity). The activity cages were distinct from the rats' home cages in several ways: the bottom of the activity cage was wire mesh (instead of bedding), and aspen chips were placed beneath the wire mesh as an olfactory cue. The rats were not exposed to the test cages prior to the beginning of each experiment. Data were collected on an attached microcomputer in 10-min intervals for the 90-min duration. Testing was always conducted between $1100 \mathrm{~h}$ and $1600 \mathrm{~h}$.

\section{Yohimbine Conditioning}

For 10 consecutive days, rats were placed in the activity cages immediately after a yohimbine injection $(n=8 ; 2 \mathrm{mg} /$ kg, i.p., dissolved in water; Sigma, St Louis, MO) or water injection $(n=8 ; 1 \mathrm{ml} / \mathrm{kg}$, i.p.). This yohimbine dose is known to be aversive in several rat behavioral paradigms (Chopin et al, 1986; File, 1986; Bhattacharya et al, 1997). The injections were always given in the testing room. Rats remained in the activity cages for $90 \mathrm{~min}$, and then were returned to their home cages. Four days after the 10-day treatment, the rats were again placed in the activity cages following only a mock injection; no drug or water was administered. Immediately following $90 \mathrm{~min}$ in the activity cages, the rats were perfused, and the brains were immunocytochemically processed for detection of the immediate-early gene product Fos (process described below). In a separate home-cage control experiment, rats ( $n=2$ per group) were treated with either yohimbine $(2 \mathrm{mg} /$ $\mathrm{kg}$, i.p.) or water for 10 days and were immediately returned to their home cages after the injection each day. Four days after this treatment, the rats were taken directly from their home cages to be perfused, and their brains were examined for Fos expression. Fos is an immediate-early gene product which, after forming a heterodimer with the products of other immediate-early genes, binds to the AP-1 promoter site in DNA, regulating transcription of various target genes. The basal level of Fos expression is low in neurons, but is transiently increased via secondary messenger systems following stimulation (Curran and Morgan, 1987), suggesting that it can be used as a marker for neuronal activation (Robertson et al, 1991).

\section{Immunocytochemistry}

Under deep anesthesia (sodium pentobarbital; The Butler Company, Columbus, $\mathrm{OH}$ ), rats were perfused transcardially with approximately $100 \mathrm{ml}$ of $0.15 \mathrm{M}$ saline containing 100 units of heparin, followed by $500 \mathrm{ml}$ of $4 \%$ paraformaldehyde in $0.01 \mathrm{M}$ phosphate buffer (PB) ( $\mathrm{pH}$ 7.4) infused over $20 \mathrm{~min}$. The brains were removed, postfixed overnight in $4 \%$ paraformaldehyde in $\mathrm{PB}$, then placed in $30 \%$ sucrose in $0.01 \mathrm{MPB}$ for $48 \mathrm{~h}$ at $4^{\circ} \mathrm{C}$. Frozen $50-\mu \mathrm{m}$-thick sections were cut on a cryostat. Sections from pairs of experimental and control animals were always processed together in an attempt to equalize background between groups. The sections were then placed in a blocking solution composed of $0.01 \mathrm{M}$ phosphate-buffered saline (PBS) (pH 7.4) containing $10 \%$ normal goat serum (NGS) and $0.2 \%$ Triton X-100 for $30 \mathrm{~min}$. Sections were rinsed three times in PBS and are incubated with the primary Fos antibody (Ab-5, rabbit polyclonal antibody, 1:50 000; Calbiochem, San Diego, CA) at $4{ }^{\circ} \mathrm{C}$ for $48 \mathrm{~h}$. After three rinses in PBS, sections were processed using a Vectastain Elite $\mathrm{ABC}$ kit (Vector, Burlingame, CA). The sections were incubated in the biotinylated goat antirabbit secondary antibody (Vector; diluted 1:200 with PBS containing 2.5\% NGS) for $60 \mathrm{~min}$, rinsed three times with PBS, and placed in the avidin-biotin complex solution for $60 \mathrm{~min}$. After rinsing three times with PBS, the peroxidase was visualized using a Vector 3, $3^{\prime}$ diaminobenzidine (DAB) substrate kit with nickel chloride enhancement. The sections were then mounted on chrome- 
alum-coated slides, air dried, dehydrated through graded alcohols, cleared in xylene and coverslipped with Permount (Fisher Scientific, Pittsburgh, PA).

\section{Data Analysis}

Behavioral data from the activity cages were analyzed using StatView software (SAS Institute, Cary, NC). For the 10-day yohimbine treatment, a two-factor, between-within analysis of variance (ANOVA) was performed with treatment as the between-subjects factor and days as the within-subjects factor. For the conditioning (test) days, a two-factor, between-within ANOVA was performed with treatment as the between-subjects factor and time (intervals) as the within-subjects factor. For the Fos immunocytochemistry data, semiquantification of labeled cells was performed using NIH Image software for the Macintosh, linked to an Olympus BX40 microscope. Images were captured at a magnification of $\times 100$ from 14 areas of each brain: (a) prelimbic cortex, anterior; (b) prelimbic cortex, posterior; (c) infralimbic cortex; (d) ventrolateral orbital cortex; (e) agranular insular cortex; (f) cingulate cortex, anterior; (g) cingulate cortex, posterior; (h) nucleus accumbens core; (i) nucleus accumbens shell; (j) lateral septum; (k) preoptic area; (l) basolateral amygdala; (m) central nucleus amygdala; and (n) sensorimotor cortex (Figure 1). The areas analyzed were chosen based on the regions previously implicated in our drug-conditioning tasks; however, the entire brain was scanned visually in an effort to locate novel regions of activation. We have focused the active search within these specified regions; however, the analysis performed does not preclude the involvement of other brain regions. The area analyzed was a standard rectangle
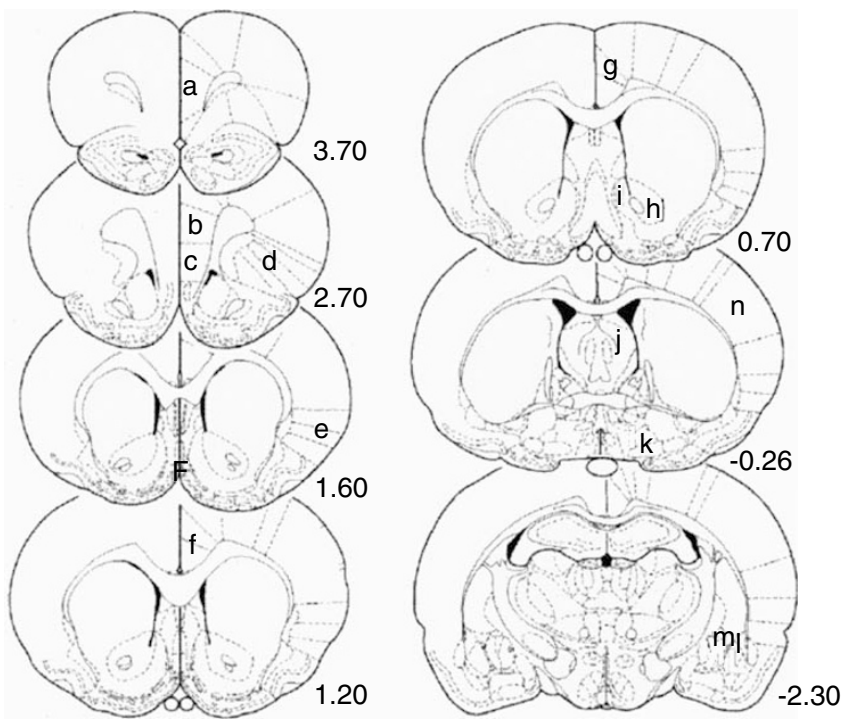

Figure I Schematic diagram of brain regions examined. Representations of coronal sections depicting specific regions that were analyzed for Fos expression are shown. Adapted with permission from Paxinos and Watson (1998). Numbers indicate distance (in $\mathrm{mm}$ ) from bregma. (a) Prelimbic cortex, anterior; (b) prelimbic cortex, posterior; (c) infralimbic cortex; (d) ventrolateral orbital cortex; (e) agranular insular cortex; (f) cingulate cortex, anterior; (g) cingulate cortex, posterior; (h) nucleus accumbens core; (i) nucleus accumbens shell; (j) lateral septum; (k) preoptic area; (I) basolateral amygdala; (m) central nucleus amygdala; (n) sensorimotor cortex.
$1060 \mu \mathrm{m} \times 790 \mu \mathrm{m}$. Each image was subjected to a consistent thresholding, reducing background in the images and increasing contrast. This thresholding criterion was set by eyeball counts of randomly selected regions from eight control and experimental slices, and was consistent across all animals. The remaining pixels (representing Fos-positive labeled cells) were counted by the computer, and entered into StatView for statistical analysis. The number of Fospositive cells in various regions were analyzed first by doing a two-way between-within ANOVA with treatment as the between-subjects factor and brain region as the withinsubjects factor. After determining whether a significant group effect or interaction was present, comparisons between individual experimental and control brain regions were performed using a one-way between-subjects ANOVA.

\section{RESULTS}

\section{Locomotor Behavior}

During the 10-day treatment period, yohimbine had a strong effect on locomotor activity (Figure $2 \mathrm{a}$ ). Horizontal activity $(\mathrm{F}(1,14)=8.609, p<0.011)$, ambulatory activity $(\mathrm{F}(1,14)=7.553, \quad p<0.0158), \quad$ and rearing activity a
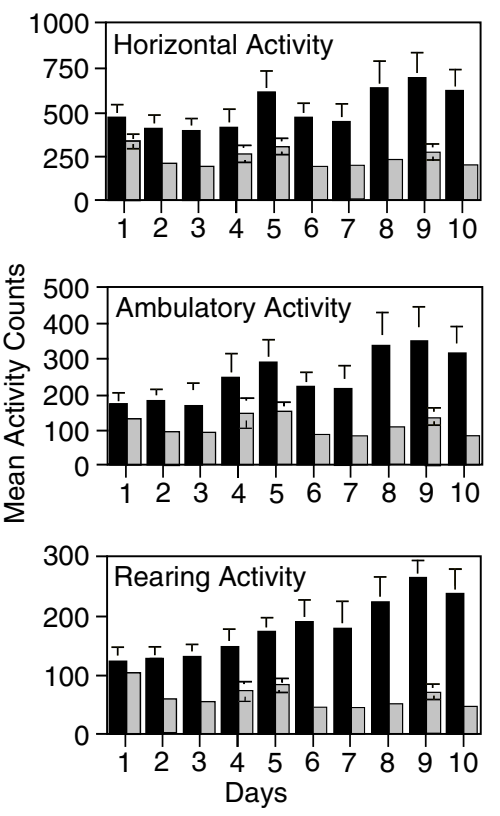

Yohimbine (2 mg/kg)

Water
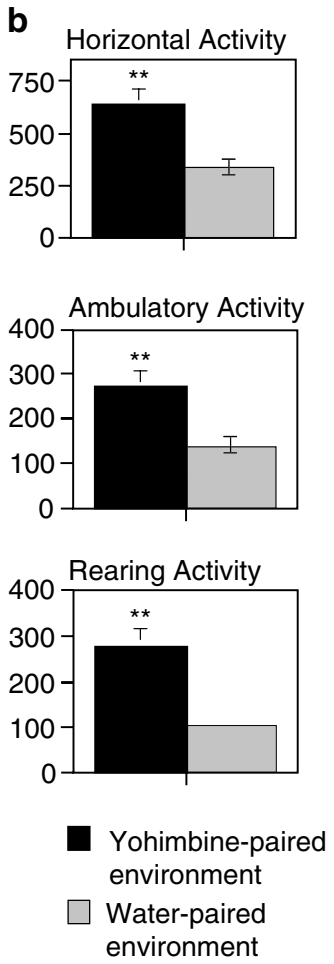

Figure 2 Behavioral data demonstrating the acute, repeated, and conditioned motor-activating effects of yohimbine. (a) Rats exposed to yohimbine $(2 \mathrm{mg} / \mathrm{kg}$, i.p.) in the activity cages during the 10-day drug treatment showed increased horizontal (shown), ambulatory, and rearing activity, as well as behavioral sensitization (see text for statistical details). (b) Rats previously treated with yohimbine showed a conditioned increase in motor activity (all three indices; see text for statistical details) when placed back into the yohimbine-paired environment 4 days after the 10-day treatment. Values represent the group mean \pm SEM. $* 2<0.05$. $* * * 0.01$, $n=8$ per group. 


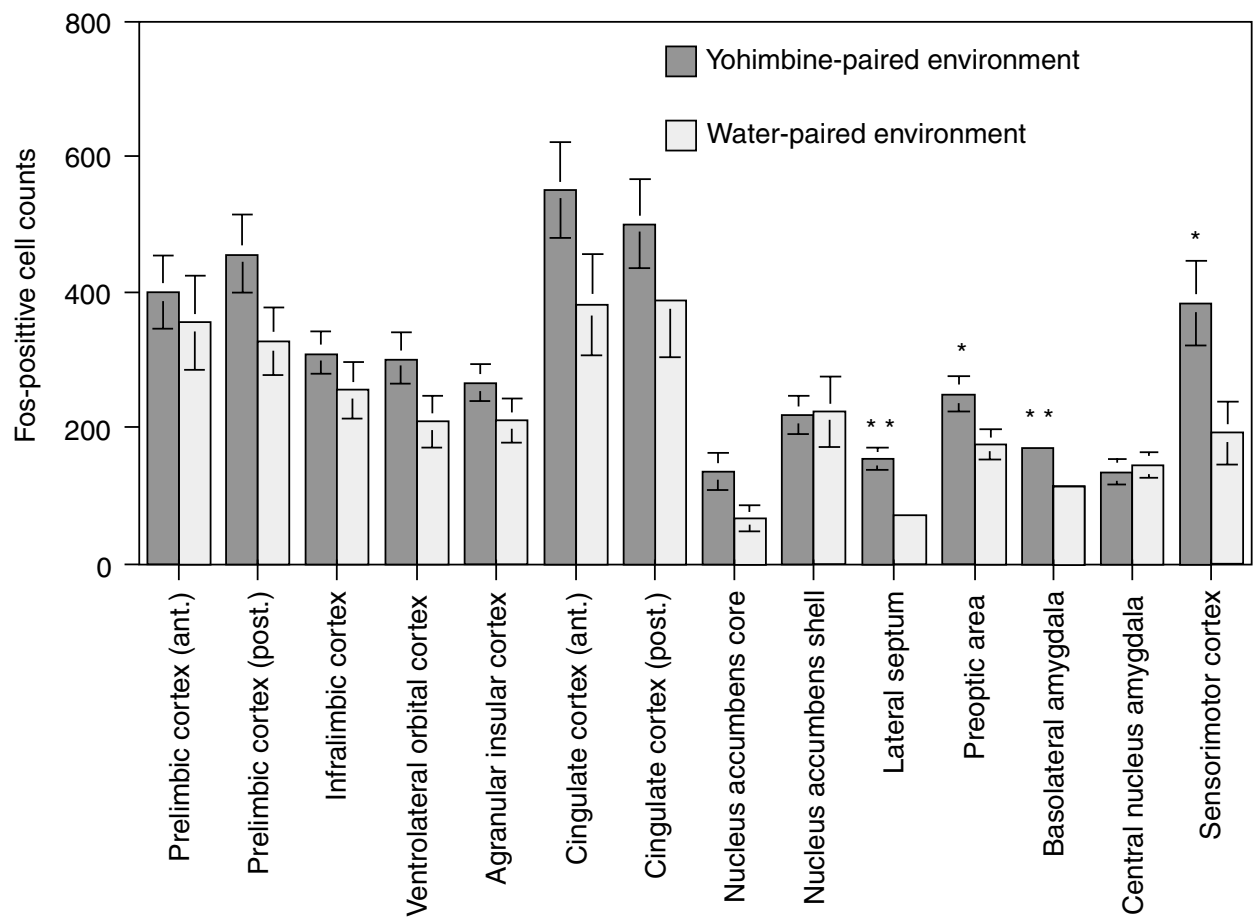

Figure 3 Fos-positive cell counts. Semiquantitative measurements of Fos-positive cells revealed significant increases in several brain regions in rats exposed to an environment where they had previously been treated with yohimbine vs rats exposed to a water-injection-paired environment. Values represent the group mean \pm SEM. $* p<0.05$. $* * * 0.01, n=8$ per group.

$(\mathrm{F}(1,14)=26.155, p<0.0003)$ were all significantly increased in the animals receiving yohimbine injections compared with those receiving water injections. Day by treatment interactions were significant for both horizontal activity $(\mathrm{F}(9,126)=2.03, p<0.0431)$ and rearing activity $(\mathrm{F}(9$, $126)=40.386, p<0.0001)$. It can be noted from Figure $2 \mathrm{a}$ that the motor response appeared to sensitize, or become progressively greater, with repeated treatments.

In addition to the direct effects of yohimbine on locomotor behavior, exposure to the yohimbine-paired environment caused conditioned increases in locomotor behavior (Figure $2 \mathrm{~b}$ ). When the rats were re-exposed to the activity cages with no yohimbine at 4 days post-treatment, horizontal activity $(\mathrm{F}(1,14)=11.239, p<0.0048)$, ambulatory activity $(\mathrm{F}(1,14)=8.891, p<0.01)$, and rearing activity $(\mathrm{F}(1,14)=14.637, p<0.002)$ were all significantly increased in the rats previously injected with yohimbine in that environment.

\section{Fos Activation}

In contrast to the results of reward conditioning experiments in our laboratory, initial examination of Fos-positive cells in the selected brain regions following exposure to the activity cage environments revealed no overall treatment effect between experimental and control groups $(\mathrm{F}(1,14)=3.581, p<0.0794)$ (Figure 3$)$. Closer analysis of individual regions revealed significant increases in basolateral amygdala $(\mathrm{F}(1,14)=9.050, p<0.0095)$, lateral septum $(\mathrm{F}(1,14)=19.903, \quad p<0.0006)$, lateral preoptic area $(\mathrm{F}(1,14)=4.630, \quad p<0.0495), \quad$ and sensorimotor cortex $(\mathrm{F}(1,14)=6.047, p<0.0277)$. Noticeably, there were no significant changes in the number of Fos-positive cells between groups in any of the prefrontal cortical regions examined. Photomicrographs from representative sections are shown in Figure 4. In the separate home-cage control experiment, no differences in Fos expression were observed between yohimbine-treated rats and the control group, and expression was at baseline control levels in both groups, indicating that the Fos effects reported above were not the result of chronic drug treatment (data not shown).

\section{DISCUSSION}

To our knowledge, this is the first report of a Fos activation profile elicited by cues associated with a presumably aversive psychoactive drug. Increases in the number of Fos-positive cells were observed in several brain regions following exposure to the yohimbine-paired environment. In particular, increased neural activity was noted in the basolateral amygdala, lateral septum, lateral preoptic area and sensorimotor cortex. Importantly, the consistent and large activation of prefrontal regions that we have noted following exposure to cues associated with reward-paired environments was not observed in this experiment. Taken together with our previous findings, the present data may aid in clarifying the roles of prefrontal cortex and the amygdala in addiction, relapse and conditioned fearful behavior.

The primary effects of yohimbine in both humans and animals are well studied. It is thought that yohimbine's antagonist action at the $\alpha-2$ adrenergic receptor causes an increase in the firing of norepinephrine-containing locus 

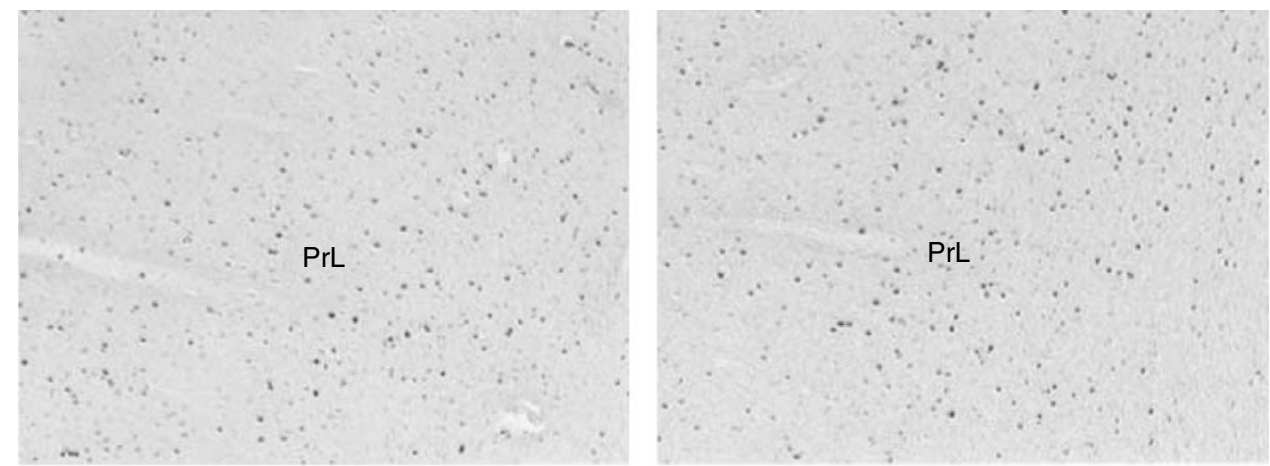

BLA

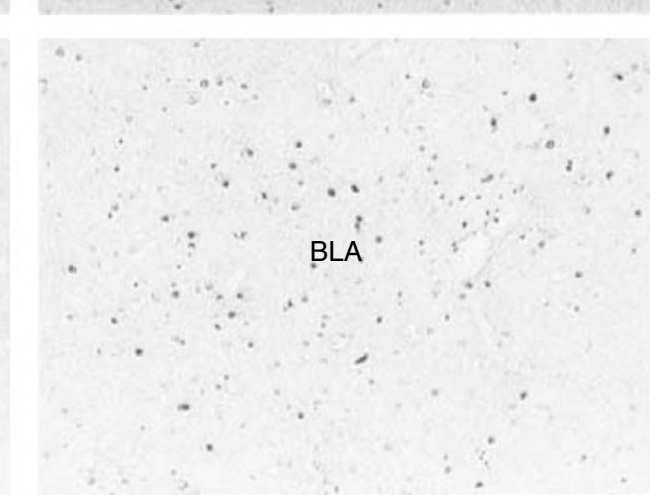

Figure 4 Photomicrographs of Fos expression in prefrontal cortex and basolateral amygdala from representative rats. The left column shows sections from rats exposed to the water-injection-paired environment; sections in the right column are from the same brain regions, but from rats exposed to the yohimbine-paired environment. × 100 magnification. PrL, prelimbic cortex; BLA, basolateral amygdala.

coeruleus neurons (Uhde et al, 1984; Pellow et al, 1985; Chopin et al, 1986). In humans, yohimbine increases self-reports of anxiety (Charney et al, 1983; Uhde et al, 1984) as well as facilitating acoustic startle reflex (Morgan et al, 1993). Animals treated with yohimbine exhibit increased anxiety and fearful behavior, including conditioned place aversion, decreased social interaction, decreased exploration and potentiated startle (Davis et al, 1979; Pellow et al, 1985; Chopin et al, 1986; File, 1986; Bhattacharya et al, 1997). Yohimbine has been shown to increase or decrease locomotor activity depending on dose, behavioral paradigm, and route of administration (Zebrowska-Lupina and Kleinrok, 1973; Chopin et al, 1986; Verleye and Bernet, 1987; Bowes et al, 1992). The present data show not only that yohimbine increased measures of locomotor activity, but also that the response appeared to sensitize over time (Figure 2a). In addition, we believe this to be the first study to demonstrate that exposure to environmental cues associated with yohimbine administration can elicit conditioned increases in locomotor behavior. These conditioned increases in locomotor behavior are comparable to the drug-induced locomotor increases and are significantly higher than locomotor behavior in control rats on day 1 of treatment, suggesting that the increases are not related to novelty effects masked by yohimbine administration. Alternatively, this increase in motor activity on the test day could be interpreted as a chronic pharmacological effect of yohimbine, a possibility that we cannot exclude based on the present data.
Perhaps more interesting is the finding that environmental cues paired with yohimbine administration elicit increases in Fos activation in discrete brain regions, and that this activation profile differs substantially from that brought about by exposure to drug-paired or palatable food-paired environments. Several previous studies have noted that acute yohimbine administration increases $c$-fos mRNA and Fos protein expression in several brain regions including cerebral cortex, locus coeruleus, central nucleus of the amygdala and bed nucleus (Gubits et al, 1989; Bing et al, 1991; Bing et al, 1992a, b; Tsujino et al, 1992; Singewald and Sharp, 2000); the effect is strongest in areas with a dense $\alpha-2$ receptor population (Tsujino et al, 1992; Singewald and Sharp, 2000). The present data revealed that mere exposure to cues associated with yohimbine administration can also elicit increased Fos expression in several brain regions.

Neural activation was particularly prominent in the basolateral amygdala following exposure to the paired environment. The amygdala has long been associated with fear and fear-related behavior. Interestingly, the basolateral amygdala may be particularly important in mediating conditioned fear responses (Fanselow and LeDoux, 1999). Basolateral amygdala lesions abolish conditioned freezing to contextual cues associated with a footshock (Maren et al, 1996), and pharmacological inactivation of the basolateral amygdala impairs memory for environments paired with a footshock (Vazdarjanova and McGaugh, 1999). Studies utilizing Fos expression have also implicated the amygdala in conditioned fear paradigms. Several groups have shown 
Table I A Comparison of Brain Regions in Which Fos-Positive Cell Counts Are Increased in Rats Conditioned to Receive Yohimbine, Morphine, Nicotine, or Chocolate as Compared to Control Groups

\begin{tabular}{lllll}
\hline Region of interest & $\begin{array}{l}\text { Yohimbine-associated } \\
\text { environment }\end{array}$ & $\begin{array}{l}\text { Morphine-associated } \\
\text { environment }^{1^{2}}\end{array}$ & $\begin{array}{l}\text { Nicotine-associated } \\
\text { environment }^{2}\end{array}$ & $\begin{array}{l}\text { Chocolate-associated } \\
\text { environment }^{\mathbf{2}}\end{array}$ \\
\hline Prelimbic cortex (ant) & - & ++ & + & + \\
Prelimbic cortex (post) & - & ++ & ++ & - \\
Infralimbic cortex & - & ++ & ++ & + \\
Ventrolateral orbital cortex & - & ++ & ++ & + \\
Agranular insular cortex & - & - & ++ & - \\
Cingulate cortex (ant) & - & + & ++ & - \\
Cingulate cortex (post) & - & - & - & - \\
Nucleus accumbens core & - & - & + & - \\
Nucleus accumbens shell & - & ++ & + & - \\
Lateral septum & ++ & - & - & - \\
Preoptic area & + & + & - & - \\
Basolateral amygdala & ++ & - & - & - \\
Central nucleus amygdala & - & - & - & - \\
Sensorimotor cortex & + & - & - \\
\hline
\end{tabular}

'Data from the morphine experiment is from Schroeder et al (2000). ${ }^{2}$ Data from the nicotine and chocolate experiments are from Schroeder et al (200I). ++, $p<0.01 ;+, p<0.05$. - , no significant change.

that cues associated with footshock increase c-fos mRNA and Fos protein expression in the amygdala (Campeau et al, 1991; Pezzone et al, 1992; Beck and Fibiger, 1995; Rosen et $a l, 1998)$. In studies that distinguished between subregions of the amygdala, basolateral amygdala activation was commonly reported; Fos expression in the central nucleus of the amygdala was present in some studies and absent in others (Pezzone et al, 1992; Beck and Fibiger, 1995). The results of our experiments concur with these previous studies, while also showing that cues associated with administration of the psychoactive and presumably anxiogenic drug yohimbine can cause increased Fos expression in the basolateral amygdala.

The absence of neural activation in the prefrontal cortex in the present experiment is particularly relevant given that, as mentioned earlier, our previous studies have shown consistent increases in Fos activation following exposure to morphine, nicotine, or chocolate-associated environments (Schroeder et al, 2000, 2001) (see Table 1 for comparisons). The behavioral specificity of this response is of critical importance, in light of the growing evidence that the prefrontal cortex is significantly involved in reward expectancy and anticipation of positive events (Gallagher et al, 1999; Leon and Shadlen, 1999). While several fear conditioning studies have previously implicated subregions of the prefrontal cortex (Beck and Fibiger, 1995; Campeau et al, 1997), others report no change in prefrontal cortical activation following exposure to footshock-associated cues (Morrow et al, 1999). To clarify this discrepancy, the present study was designed to precisely parallel our previous experiments such that the only difference between experiments was that yohimbine was administered during the treatment phase instead of a rewarding drug or palatable food reward. Under this paradigm, we found that environmental cues associated with a presumably aversive experience (yohimbine treatment) do not elicit increases in Fos expression in any regions of the prefrontal cortex. Interestingly, these data also further support our previous hypothesis that increases in neural activation in prefrontal cortex following exposure to drug-paired cues are not causally related to conditioned increases in motor responses. Most importantly, however, the contrast in profiles between these data and our previous findings strengthens the hypothesis that the prefrontal cortex is preferentially activated during drug or reward expectancy.

Several brain regions implicated in the present findings were also activated in our earlier experiments. Fos expression was increased in the preoptic area following exposure to a morphine- or chocolate-associated environment, as well as the yohimbine-paired environment, thus making it less likely to be specific for cues associated with either a positive or a negative affective valence. The number of Fos-positive cells also was increased in the lateral septum following exposure to a nicotine-associated environment and in response to the yohimbine-paired environment. At first glance, this may appear to restrict the specificity of the response of the lateral septum, but an alternative mechanism may explain the discrepancy. In addition to its reinforcing effects, nicotine has been found by some researchers to have aversive properties under some circumstances (Fudala et al, 1985; Risinger and Oakes, 1995). Likewise, the lateral septum has been implicated in aversive and defensive behaviors (Koolhaas et al, 1980; Pezzone et al, 1992), and exposure to an environment paired with a footshock induces Fos expression in lateral septum (Pezzone et al, 1992; Beck and Fibiger, 1995). Activation of the lateral septum potentially may, therefore, be specific to cues associated with aversive events. Although not activated under any of our previous paradigms, the activation of sensorimotor cortex described in the present findings is not entirely unexpected, given that several fear conditioning studies have shown parietal cortex Fos expression (Pezzone et al, 1992; Beck and Fibiger, 1995; Radulovic et al, 1998). This activation may reflect vigilance or arousal caused by exposure to the aversive-paired environment.

Recognizing environmental cues that predict aversive events or signal potentially beneficial rewards in the environment is an important adaptive behavior. Recognition of environmental cues associated with drug adminis- 
tration, however, may lead to cravings and relapse in former addicts. Understanding the brain regions and pathways that underlie conditioned behavior is, therefore, of critical importance in treating drug addiction. The present findings may help to clarify the role of prefrontal cortex and amygdala structures often implicated in drug-associated conditioning. Definition of the neurochemical substrates involved in these phenomena and of the interconnections between the implicated brain regions awaits further study.

\section{ACKNOWLEDGEMENTS}

This research was supported by Grant DA04788 from the National Institute on Drug Abuse and National Research Service Award (NRSA) DA05929.

\section{REFERENCES}

Beck CH, Fibiger HC (1995). Conditioned fear-induced changes in behavior and in the expression of the immediate early gene $c$-fos: With and without diazepam pretreatment. J Neurosci 15: 709720.

Bhattacharya SK, Satyan KS, Chakrabarti A (1997). Anxiogenic action of caffeine: an experimental study in rats. J Psychopharmacol 11: 219-224.

Bing G, Chen S, Zhang Y, Hillman D, Stone EA (1992a). Noradrenergic-induced expression of $c$-fos in rat cortex: neuronal localization. Neurosci Lett 140: 260-264.

Bing G, Stone EA, Zhang Y, Filer D (1992b). Immunohistochemical studies of noradrenergic-induced expression of $c$-fos in the rat CNS. Brain Res 592: 57-62.

Bing GY, Filer D, Miller JC, Stone EA (1991). Noradrenergic activation of immediate early genes in rat cerebral cortex. Brain Res Mol Brain Res 11: 43-46.

Bowes MP, Peters RH, Kernan Jr WJ, Hopper DL (1992). Effects of yohimbine and idazoxan on motor behaviors in male rats. Pharmacol Biochem Behav 41: 707-713.

Brown EE, Robertson GS, Fibiger HC (1992). Evidence for conditional neuronal activation following exposure to a cocaine-paired environment: Role of forebrain limbic structures. J Neurosci 12: 4112-4121.

Campeau S, Falls WA, Cullinan WE, Helmreich DL, Davis M, Watson SJ (1997). Elicitation and reduction of fear: behavioural and neuroendocrine indices and brain induction of the immediate-early gene $c$-fos. Neuroscience 78: 1087-1104.

Campeau S, Hayward MD, Hope BT, Rosen JB, Nestler EJ, Davis M (1991). Induction of the $c$-fos proto-oncogene in rat amygdala during unconditioned and conditioned fear. Brain Res 565: 349352.

Charney DS, Heninger GR, Redmond Jr DE (1983). Yohimbine induced anxiety and increased noradrenergic function in humans: Effects of diazepam and clonidine. Life Sci 33: 19-29.

Childress AR, Mozley PD, McElgin W, Fitzgerald J, Reivich M, O’Brien CP (1999). Limbic activation during cue-induced cocaine craving. Am J Psychiatry 156: 11-18.

Chopin P, Pellow S, File SE (1986). The effects of yohimbine on exploratory and locomotor behaviour are attributable to its effects at noradrenaline and not at benzodiazepine receptors. Neuropharmacology 25: 53-57.

Ciccocioppo R, Sanna PP, Weiss F (2001). Cocaine-predictive stimulus induces drug-seeking behavior and neural activation in limbic brain regions after multiple months of abstinence: reversal by D(1) antagonists. Proc Natl Acad Sci USA 98: 1976-1981.
Curran T, Morgan JI (1987). Memories of fos. Bioessays 7: 255-258. Davis M, Redmond Jr DE, Baraban JM (1979). Noradrenergic agonists and antagonists: effects on conditioned fear as measured by the potentiated startle paradigm. Psychopharmacology (Berl) 65: 111-118.

Fanselow MS, LeDoux JE (1999). Why we think plasticity underlying Pavlovian fear conditioning occurs in the basolateral amygdala . Neuron 23: 229-232.

File SE (1986). Aversive and appetitive properties of anxiogenic and anxiolytic agents. Behav Brain Res 21: 189-194.

Franklin TR, Druhan JP (2000). Expression of Fos-related antigens in the nucleus accumbens and associated regions following exposure to a cocaine-paired environment. Eur J Neurosci 12: 2097-2106.

Fudala PJ, Teoh KW, Iwamoto ET (1985). Pharmacologic characterization of nicotine-induced conditioned place preference. Pharmacol Biochem Behav 22: 237-241.

Gallagher M, McMahan RW, Schoenbaum G (1999). Orbitofrontal cortex and representation of incentive value in associative learning. J Neurosci 19: 6610-6614.

Grant S, London ED, Newlin DB, Villemagne VL, Liu X, Contoreggi $C$ et al (1996). Activation of memory circuits during cueelicited cocaine craving. Proc Natl Acad Sci USA 93: 12040 12045.

Gubits RM, Smith TM, Fairhurst JL, Yu H (1989). Adrenergic receptors mediate changes in $c$-fos mRNA levels in brain. Brain Res Mol Brain Res 6: 39-45.

Kandel ER, Kupfermann I, Iverson S (2000). Learning and memory. In: Kandel ER, Schwartz JH, Jessell TM (eds). Principles of Neural Science, 4th edn. McGraw-Hill Health Professions Division: New York, pp 1227-1246.

Koolhaas JM, Schuurman T, Wiepkema PR (1980). The organization of intraspecific agonistic behaviour in the rat. Prog Neurobiol 15: 247-268.

LeDoux JE (2000). Emotion circuits in the brain. Annu Rev Neurosci 23: 155-184.

Leon MI, Shadlen MN (1999). Effect of expected reward magnitude on the response of neurons in the dorsolateral prefrontal cortex of the macaque. Neuron 24: 415-425.

Maas LC, Lukas SE, Kaufman MJ, Weiss RD, Daniels SL, Rogers VW et al (1998). Functional magnetic resonance imaging of human brain activation during cue-induced cocaine craving. Am J Psychiatry 155: 124-126.

Maren S, Aharonov G, Fanselow MS (1996). Retrograde abolition of conditional fear after excitotoxic lesions in the basolateral amygdala of rats: absence of a temporal gradient. Behav Neurosci 110: 718-726.

Morgan 3rd CA, Southwick SM, Grillon C, Davis M, Krystal JH, Charney DS (1993). Yohimbine-facilitated acoustic startle reflex in humans. Psychopharmacology (Berl) 110: 342-346.

Morrow BA, Elsworth JD, Inglis FM, Roth RH (1999). An antisense oligonucleotide reverses the footshock-induced expression of fos in the rat medial prefrontal cortex and the subsequent expression of conditioned fear-induced immobility. J Neurosci 19: $5666-5673$.

O'Brien CP, Childress AR, Ehrman R, Robbins SJ (1998). Conditioning factors in drug abuse: can they explain compulsion? J Psychopharmacol 12: 15-22.

Paxinos G, Watson C (1998). The Rat Brain in Stereotaxic Coordinates, 4th edn. Academic Press: San Diego.

Pellow S, Chopin P, File SE (1985). Are the anxiogenic effects of yohimbine mediated by its action at benzodiazepine receptors? Neurosci Lett 55: 5-9.

Pezzone MA, Lee WS, Hoffman GE, Rabin BS (1992). Induction of $c$-fos immunoreactivity in the rat forebrain by conditioned and unconditioned aversive stimuli. Brain Res 597: 41-50.

Radulovic J, Kammermeier J, Spiess J (1998). Relationship between fos production and classical fear conditioning: effects of novelty, 
latent inhibition, and unconditioned stimulus preexposure. $J$ Neurosci 18: 7452-7461.

Risinger FO, Oakes RA (1995). Nicotine-induced conditioned place preference and conditioned place aversion in mice. Pharmacol Biochem Behav 51: 457-461.

Robertson HA, Paul ML, Moratalla R, Graybiel AM (1991). Expression of the immediate early gene $c$-fos in basal ganglia: induction by dopaminergic drugs. Can J Neurol Sci 18: 380-383.

Rosen JB, Fanselow MS, Young SL, Sitcoske M, Maren S (1998). Immediate-early gene expression in the amygdala following footshock stress and contextual fear conditioning. Brain Res 796: 132-142.

Sanger DJ (1988). Behavioural effects of the alpha 2-adrenoceptor antagonists idazoxan and yohimbine in rats: comparisons with amphetamine. Psychopharmacology (Berl) 96: 243-249.

Schroeder BE, Binzak JM, Kelley AE (2001). A common profile of prefrontal cortical activation following exposure to nicotine- or chocolate-associated contextual cues. Neuroscience 105: 535-545.

Schroeder BE, Holahan MR, Landry CF, Kelley AE (2000). Morphine-associated environmental cues elicit conditioned gene expression. Synapse 37: 146-158.

Self DW, Nestler EJ (1998). Relapse to drug-seeking: neural and molecular mechanisms. Drug Alcohol Depend 51: 49-60.
Singewald N, Sharp T (2000). Neuroanatomical targets of anxiogenic drugs in the hindbrain as revealed by Fos immunocytochemistry. Neuroscience 98: 759-770.

Tsujino T, Sano H, Kubota Y, Hsieh ST, Miyajima T, Saito K et al (1992). Expression of Fos-like immunoreactivity by yohimbine and clonidine in the rat brain. Eur J Pharmacol 226: 69-78.

Uhde TW, Boulenger JP, Post RM, Siever LJ, Vittone BJ, Jimerson DC et al (1984). Fear and anxiety: relationship to noradrenergic function. Psychopathology 17: 8-23.

Vazdarjanova A, McGaugh JL (1999). Basolateral amygdala is involved in modulating consolidation of memory for classical fear conditioning. J Neurosci 19: 6615-6622.

Verleye M, Bernet F (1987). Behavioral effects of intrahippocampal injections of clonidine, yohimbine and salbutamol in the rat. Pharmacol Biochem Behav 26: 421-424.

Wikler A, Pescor FT (1967). Classical conditioning of a morphine abstinence phenomenon, reinforcement of opioid-drinking behavior and 'relapse' in morphine-addicted rats. Psychopharmacologia 10: 255-284.

Zebrowska-Lupina I, Kleinrok Z (1973). Behavioural effects of yohimbine administered intraventricularly in the rat. Psychopharmacologia 33: 267-275. 